\title{
FLORA OF THE ISLETS NEAR PAKOŠTANE (DALMATIA, CROATIA)
}

\author{
Marija PandžA ${ }^{1} \&$ Milenko Milović ${ }^{2}$ \\ ${ }^{1}$ Primary School Murterski škoji, Put škole 8, HR-22243 Murter, Croatia \\ (E-mail: marija.pandza@si.t-com.hr) \\ ${ }^{2}$ Medical and Chemical School, Ante Šupuk Street, HR-22000 Šibenik, Croatia \\ (E-mail: milenko.milovic@si.t-com.hr)
}

Pandža, M. \& Milović, M.: Flora of the islets near Pakoštane (Dalmatia, Croatia). Nat. Croat., Vol. 24, No. 1, 19-35, 2015, Zagreb.

During 2004 and 2012 field research into the flora of the five uninhabited islets near Pakoštane, Dalmatia, South Croatia was carried out: Babuljaš, Veliki Školj, St Justina, Mali and Veliki Žavinac. Altogether, 257 vascular flora taxa were recorded. The flora of the islet Veliki Školj had 159 taxa, that of St Justina 118, Veliki Žavinac 115, Mali Žavinac 105 and Babuljaš 95.

The largest number of taxa were found in the families Fabaceae (40 taxa; 15.56\%), Poaceae (36 taxa; $14.00 \%$ ) and Asteraceae s.l. (31 taxa; $12.06 \%$ ). Regarding life forms, therophytes prevailed ( $49.42 \%)$. The chorological spectrum highlights a clear dominance of Mediterranean taxa (50.19\%). Their percentage contribution did not significantly differ among the islets (47.62-53.04\%).

There were four endemic, eight endangered and eight strictly protected plant taxa. No neophytes were found except Conyza sumatrensis which was observed on the islet of St Justina.

Key words: diversity, vascular flora, small islets, Adriatic Sea, Dalmatia, Croatia

Pandža, M. \& Milović, M.: Flora otočića ispred Pakoštana (Dalmacija, Hrvatska). Nat. Croat., Vol. 24, No. 1, 19-35, 2015, Zagreb.

Tijekom 2004. i 2012. provedena su terenska istraživanja flore na pet nenaseljenih otočića ispred Pakoštana: Babuljaš, Veliki Školj, Sveta Justina, Mali i Veliki Žavinac. Utvrđeno je ukupno 257 svojti vaskularne flore. Flora Velikog Školja ima 159 svojti, Sv. Justine 118, Velikog Žavinca 115, Malog Žavinca 105 i Babuljaša 95 svojti.

Porodice s najvećim brojem svojti su Fabaceae (40 svojti; 15,56 \%), Poaceae (36 svojti; 14,00\%) i Asteraceae s.l. (31 svojta; $12,06 \%$ ). Terofiti su najzastupljeniji životni oblik (49,42\%), a među flornim elementima najzastupljenije su mediteranske biljke (50,19\%). Biljke mediteranskog rasprostranjenja približno su jednako (47,62-53,04\%) zastupljene na svim otočićima. Zabilježena su četiri endema, osam ugroženih te osam strogo zaštićenih biljaka. Od neofita, na Sv. Justini je zabilježena samo vrsta Conyza sumatrensis dok na ostalim otočićima neofita nije bilo.

Ključne riječi: raznolikost, vaskularna flora, otočići, Jadransko more, Dalmacija, Hrvatska

\section{INTRODUCTION}

According to Duplančić Leder et al. (2004) in the Croatian part of the Adriatic Sea there are 79 islands (area $\left.>1 \mathrm{~km}^{2}\right), 525$ islets $\left(0,01-1 \mathrm{~km}^{2}\right)$ and 642 rocks and rocks awash $\left(<0,01 \mathrm{~km}^{2}\right)$. In general, the larger and inhabited islands of the Adriatic archipelago are botanically well explored and recognized as one of the major centers of plant diversity in Croatia (NiкоLıć et al., 2008, 2014). But there is still a significant number of uninhabi- 
ted islets and reefs where the vascular flora, apart from a few exceptions (Hećrmović, 1982; PAndžA, 2002; PAvletić, 1983; JAspricA \& Ruščić, 2013; SKelin et al., 2014), is practically unexplored. There are five islets off the village of Pakoštane (Zadar archipelago, north Dalmatia): Babuljaš, Veliki Školj, St Justina, Mali Žavinac and Veliki Žavinac (Fig. 1) whose flora has never been investigated before. The aim of this study is to investigate the vascular flora of these five islets and to compare it with other small uninhabited islets of the Adriatic archipelago.

\section{STUDY AREA}

The islets which are the subject of this study are part of the Zadar archipelago, which belongs to the group of North Dalmatian islands (STRAžıčıć, 1987), Fig. 1. They are situated off the village of Pakoštane at distances from $260 \mathrm{~m}$ (St Justina) to $570 \mathrm{~m}$ (Veli Skoj) from the nearest shore (Fig. 1). Four of them are considerably smaller than $1 \mathrm{~km}^{2}$ which puts them into the islets category: Veliki Školj $\left(0.0549 \mathrm{~km}^{2}\right)$, St Justina $\left(0.0125 \mathrm{~km}^{2}\right)$, Žavinac Mali $\left(0.0115 \mathrm{~km}^{2}\right)$ and Žavinac Veliki $\left(0.0277 \mathrm{~km}^{2}\right)$. Babuljaš $\left(0.0078 \mathrm{~km}^{2}\right)$ is included

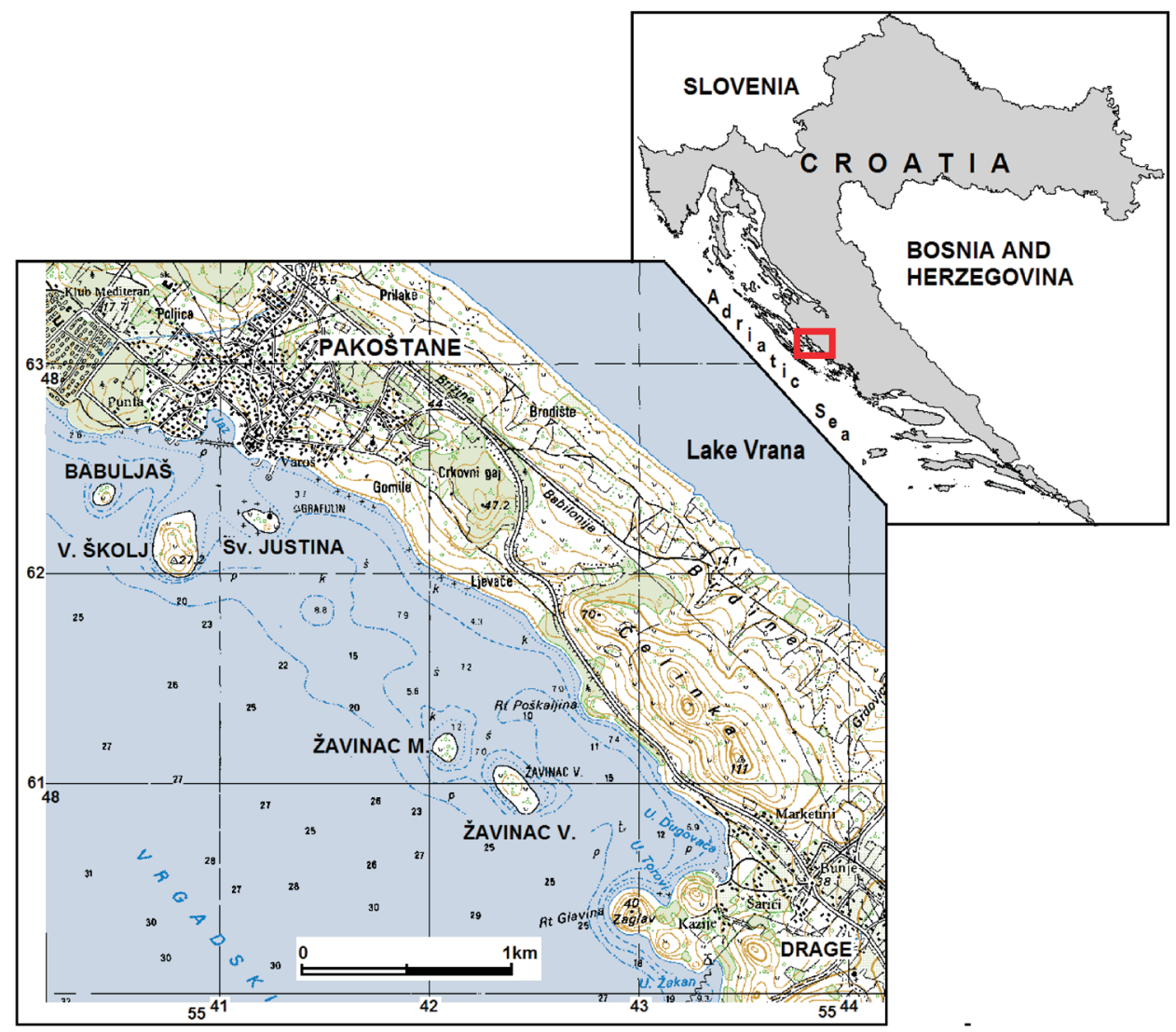

Fig. 1. Geographical position of the studied islets. 
into the reefs category (Duplančić Leder et al., 2004). All of them are uninhabited and are included into the group of small, occasionally inhabited or uninhabited islands and islets - MPNNOo, the Area of Special State Concern (Anonymous, 2007). The National Programme for Protection and use of MPNNOo was adopted in order to protect them from inappropriate and unplanned management.

Although they are located very close to the mainland, there is little human impact on the investigated islands. On St Justina people occasionally pay visits to the small church and tend the small olive grove that has been recently planted and the islet of Veliki Školj is used for goat grazing.

According to Köppen classification, they are within the $\mathrm{C}_{\mathrm{sa}}$ climate zone (BERTOVIĆ, 1975; ŠEGOTA \& FILIPČIĆ, 2003) characterized by hot summers with the average monthly temperature of $\geq 22{ }^{\circ} \mathrm{C}$. The nearest weather station in Biograd reported the average annual precipitation of $815.4 \mathrm{~mm}$ and the average annual air temperature was $15^{\circ} \mathrm{C}$ for the period from $1981-2010$. The lowest precipitation values were recorded in July, $23.5 \mathrm{~mm}$ average and the highest in the period from October to March. These are typical maritime precipitation regime characteristics (Penzar \& Penzar, 1979/80; 1981). During summer, vegetation practically stops growing due to harsh droughts.

These islets are situated within the Eu-Mediterranean belt of evergreen vegetation of the Fraxino orni-Quercion ilicis Biondi, Casavecchia \& Gigante 2013 alliance while the coastal area belongs to the Plantagini-Limonietum cancellati Horvatić (1934) 1939) association. The muddy soil in the north-west of the St. Justina islet is covered by the Puccinellio festuciformis-Salicornietum fruticosae (Br.-B1. 1928) Géhu 1967 association (Croatian National Classification of Habitats, NCH - F.1.1.3.1 - endangered and protected habitat, Topić \& Vukelić, 2009) which is mentioned in Directive 92/43 EEC (Anonymous, 1992). Mosaics of low bushes and clearings with dry grassland vegetation can be seen on every islet.

\section{METHODS}

Field work on flora inventory was performed in 2004 and 2012. Identification of plant taxa was carried out using the standard floristic literature: BonNIER (1911-1935), Fiori (1923-1929), HayeK (1924-1933), Hegi (1936-1987), Tutin et al. (1968-1980, 1993), Horvatić \& Trinajstić (1967-1981), Trinajstić (1975-1986), JAVORKA \&CsAPODY (1975), Pignatti (1982) and Domac (1994). The nomenclature of plant taxa follows Nikolić (2014a).

The list of families, genera, species and subspecies is given in alphabetical order and is organised within higher system units. Designations for islets, life forms, chorological type (geoelement), threatened and protected status were provided for taxa in the flora list.

Based on the life form classification of RAUNKIAER (1934), biological form was verified in the field and denoted according to categories reported in Horvat (1949) and Pignatti (1982): T - Therophytes, G - Geophytes, H - Hemicryptophytes, Ch - Chamaephytes and P - Phanerophytes.

The division of the plants into floral elements and lower categories was made according to Horvatić (1963) and Horvatić et al. (1967-1968). In the list of flora, each species name is followed by the floral element and marked as follows: 


\section{MEDITERRANEAN (MED)}

\subsection{Circum-Mediterranean (CM)}

1.2. West Mediterranean (ZM)

1.3. East Mediterranean (IM)

1.4. Illyrian Mediterranean plants

A) Illyrian South European (ILJEU)

B) Illyrian Adriatic plants

a) Illyrian Adriatic Endemic plants (ILJAE)

b) Illyrian Apennine plants (ILAP)

\subsection{Mediterranean Atlantic (MA)}

1.6. European Mediterranean (EUM)

1.7. Mediterranean Pontic (MP)

\section{SOUTH EUROPEAN (SEU)}

2.1. South European Mediterannean plants (JEUM)

2.2. South European Pontic (JEUP)

2.3. South European-Atlantic (JT)

3. ATLANTIC PLANTS (AN)

4. EAST EUROPEAN-PONTIC (IEP)

5. EUROPEAN (EF)

6. EUROASIATIC (EAF)

\section{CIRCUM-HOLARCTIC (CIRC)}

8. WIDESPREAD PLANTS (ک̌R)

\section{CULTURAL AND ADVENTIVE PLANTS (N)}

In the list of flora the islets are marked by numbers given after the floral element: 1 - Babuljaš, 2 - Veliki Školj, 3 - St. Justina, 4 - Žavinac Mali and 5 - Žavinac Veliki. Abbreviation "end" in the list of flora stands for endemic taxa, as classified by NiкоLIĆ (2014a).

Species from the Red Book List of the Vascular Flora of Croatia (Nikolić \& Topić, 2005; Niкоцić 2014b) are marked by proper abbreviations showing the degree of each species endangerment: Endangered (EN), Vulnerable (VU), Near Threatened (NT), Laest Concern (LC) and Data Deficient (DD).

Taxa protected by the Nature Protection Act (Anonymous, 2013a) and listed in the Ordinance on Designating Wild Taxa Protected (Anonymous, 2013b) are denoted as "spr"-strictly protected.

In the floristic list, habitats are given after the phytogeographical element and marked by letters as follows: $\mathrm{a}$ - macchia and garrigue, $\mathrm{b}$ - Aleppo pine forests; $\mathrm{c}-$ dry grasslands; d - ruderal habitats; e - muddy and salty soils; f - rocky sea shores; $\mathrm{g}$ - rocks and cracks in the walls; $\mathrm{h}$ - parasites on the plants. 


\section{RESULTS}

\section{Floristic list}

\section{PTERIDOPHYTA}

\section{Aspleniaceae}

$\mathrm{H} \quad$ Asplenium trichomanes L.; ŠR; 2; g

\section{SPERMATOPHYTA-CONIFEROPHYTINA}

\section{Cupressaceae}

P Cupressus sempervirens L.; IM; 1, 2, 3; b

P Juniperus oxycedrus L. ssp. macrocarpa (Sibth. et Sm.) Ball; CM; 1, 5; a

P J. oxycedrus L. ssp. oxycedrus; CM; 1, 2, 4; a

P J. phoenicea L.; CM; 1 ; a

\section{Pinaceae}

P Pinus halepensis Mill.; CM; 2, 3, 5; b

\section{SPERMATOPHYTA-MAGNOLIOPHYTINA}

\section{MAGNOLIATAE}

\section{Anacardiaceae}

P Pistacia lentiscus L.; CM; 1, 2, 3, 5; a, b

P P. terebinthus L.; CM; $4 ; \mathrm{a}, \mathrm{b}$

Apiaceae

Ch Crithmum maritimum L.; MA; 1, 2, 3, 4, 5; f

$\mathrm{H}$ Eryngium amethystinum L.; ILJEU; 5; c

$\mathrm{T}$ Torilis arvensis (Huds.) Link; JEUM; 4, 5; c, d

T T. nodosa (L.) Gaertn.; MA; 1, 2, 3, 5; c, d

\section{Asclepiadaceae}

H Vincetoxicum hirundinaria Medik ssp. adriaticum (Beck) Markgr.; ILJAE; 4, 5; c; end; spr; LC

\section{Asteraceae}

Ch Artemisia caerulescens L.; ILAP; 3; e

T Bombycilaena erecta (L.) Smoljan.; JEUP; 2; c, d

$\mathrm{H} \quad$ Carduus micropterus (Borbás) Teyber ssp. micropterus; ILJAE; 1, 2, 3; c; end; spr

$\mathrm{H} \quad$ C. pycnocephalus L. ssp. pycnocephalus; CM; 1, 2, 3, 4; d; DD

$\mathrm{H} \quad$ Carlina corymbosa L.; CM; 2, 3, 4, 5; c

$\mathrm{H}$ Cirsium vulgare (Savi) Ten.; EAF; 3 ; c, d

$\mathrm{T} \quad$ Conyza sumatrensis (Retz.) E. Walker; $\mathbf{N} ; 3 ; \mathrm{d}$

$\mathrm{H} \quad$ Dittrichia viscosa (L.) Greuter; CM; 5; f

Ch Helichrysum italicum (Roth) G. Don; CM; 2, 5; a, c, d, f

Ch Inula crithmoides L.; MA; 3, 4, 5; e, f

$\mathrm{H}$ Onopordum illyricum L.; CM; 1, 2, 3; c, d

$\mathrm{H}$ Picnomon acarna (L.) Cass.; CM; 2; c

$\mathrm{T} \quad$ Senecio vulgaris L.; ŠR; 5 ; $\mathrm{d}$

\section{Boraginaceae}

T Myosotis arvensis (L.) Hill; EAF; 2, 3, 4, 5; c, d 


\section{Brassicaceae}

Ch Aethionema saxatile (L.) R.Br.; JEUM; 5; a, b

$\mathrm{H} \quad$ Arabis hirsuta (L.) Scop.; ŠR; 4, 5; c, d

Ch Aurinia sinuata (L.) Griseb.; ILAP; 1, 2; c, d, g; end; spr

T Biscutella cichoriifolia Loisel.; JEUM; $1 ; \mathrm{g}$

T Capsella rubella Reut. ; CM; 1, 2, 3, 5; d

$\mathrm{T} \quad$ Cardamine hirsuta L.; ŠR; 2 ; c, d

T Clypeola jonthlaspi L.; CM; 2 ; C

H Diplotaxis tenuifolia (L.) DC.; ŠR; 3 ; d

$\mathrm{H} \quad$ Lepidium graminifolium L. ssp. suffruticosum (L.) P.Monts.; JEUP; 1, 3, 4; d

$\mathrm{T} \quad$ Sinapis arvensis L.; ŠR; 2 ; d

T Sisymbrium officinale (L.) Scop.; ŠR; 1, 2, 3, 4, 5; d

\section{Campanulaceae}

$\mathrm{H}$ Campanula rapunculus L.; EAF; 5; C

\section{Caprifoliaceae}

P Lonicera implexa Aiton; CM; 4, 5; a

\section{Caryophyllaceae}

T Arenaria leptoclados (Reichenb.) Guss.; EAF; 1, 2, 3, 4, 5; b, c

T A. serpillyfolia L.; ŠR; 1, 2, 4, 5; c

T Cerastium brachypetalum Pers. ssp. roeseri (Boiss. et Heldr.) Nyman; JT; 5; c

T C. pumilum Curtis ssp. glutinosum (Fries) Jalas; ŠR; 2, 3, 4, 5; C

T C. semidecandrum L.; JEUP; 2, 5; c

$\mathrm{T} \quad$ Herniaria glabra L.; EAF; 2; f

$\mathrm{H} \quad$ H. incana Lam.; JEUM; 5; c, d

T Minuartia hybrida (Vill.) Schischkin; EAF; 2; c

H Petrorhagia saxifraga (L.) Link; JEUM; 2, 4, 5; c

T Polycarpon tetraphyllum (L.) L.; ŠR; $1,2,4$; c, d, f

T Sagina maritima G.Don; ŠR; $1,2,5 ; \mathrm{f}$

$\mathrm{H} \quad$ Silene latifolia Poir. ssp. alba (Mill.) Greuter et Bourdet; EAF; 1, 2, 3, 4; d

$\mathrm{H} \quad$ S. vulgaris (Moench) Garcke ssp. angustifolia Hayek; JEUM; 1, 2, 3, 4, 5; a, c, f

$\mathrm{T} \quad$ Spergularia salina J.Presl et C.Presl; SR; $3 ; \mathrm{f}$

$\mathrm{T} \quad$ Stellaria media (L.) Vill.; ŠR; 1, 2, 5; d

\section{Chenopodiaceae}

Ch Arthrocnemum fruticosum (L.) Moq.; JEUM; 3; e

Ch A. macrostachyum (Moric) C. Koch; JEUM; 1, 3, 4, 5; e, f

$\mathrm{T}$ Atriplex prostrata Boucher ex DC in Lam. et DC.; ŠR; 1, 2, 3, 4; f

$\mathrm{H} \quad$ Beta vulgaris L. ssp. maritima (L.) Arcang.; MA; 3, 4; f

Ch Camphorosma monspeliaca L.; CM; 1, 3; c

T Chenopodium album L.; ŠR; $1,2,5$; d

T Ch. strictum Roth; ŠR; 1 ; d

Ch Halimione portulacoides (L.) Aellen; CIRC; 3; e

\section{Cichoriaceae}

G Aetheorhiza bulbosa (L.) Cass.; CM; 1, 2, 4, 5; a, b, c, f

T Crepis neglecta L.; EUM; 3, 4, 5; c

T C. rubra L.; IM; 1, 3; c

T C. sancta (L.) Babc.; IM; 1, 2; c, d

T C. zacintha (L.) Babc.; CM; 2; c 
T Hedypnois cretica (L.) Dum.Cours.; CM; 1, 2, 4; c

$\mathrm{H} \quad$ Hieracium piloselloides Vill. ILJEU; 3; c

H Lactuca serriola L.; ŠR; 3; d

$\mathrm{H} \quad$ Leontodon tuberosus L.; CM; 2; a, c

$\mathrm{H}$ Reichardia picroides (L.) Roth.; CM; 1, 2, 3, 4, 5; a, c, f

$\mathrm{T} \quad$ Rhagadiolus stellatus (L.) Gaertn.; CM; 2, 3; b, c, d

$\mathrm{H}$ Scolymus hispanicus L.; CM; 2, 3; d

$\mathrm{H}$ Scorzonera villosa Scop.; ILJEU; 2; c

T Sonchus asper (L.) Hill ssp. glaucescens (Jord.) Ball; CM; 2, 3, 5; d, f

T S. tenerrimus L.; CM; 1, 3, 5; d

$\mathrm{H}$ Tragopogon dubius Scop.; JEUP; 3; c

$\mathrm{H} \quad$ T. porrifolius L.; CM; 3; c

T Urospermum picroides (L.) Scop. ex F.W. Schmidt; CM; 2, 3, 4, 5; a, c

\section{Clusiaceae}

$\mathrm{H} \quad$ Hypericum perforatum L. ssp. veronense (Schrank) H. Lindb.; JEUM; 1, 3, 4, 5; c

\section{Convolvulaceae}

G Convolvulus arvensis L.; ŠR; 3, 4; c, d

\section{Crassulaceae}

Ch Sedum acre L.; ŠR; 2, 3, 4, 5; c, g

T S. rubens L.; JEUM; 2, 5; C

\section{Cuscutaceae}

T Cuscuta epithymum (L.) L.; ŠR; 2, 4, 5; h

\section{Euphorbiaceae}

T Euphorbia helioscopia L.; ŠR; 1, 2, 3; d

T E. peplus L.; ŠR; 2, 3; d

Ch E. pinea L.; CM; 1, 4; $\mathrm{f}$

Ch E. spinosa L.; CM; 2; c, g

$\mathrm{T}$ Mercurialis annua L.; ŠR; 1, 2, 3; b, d, g

\section{Fabaceae}

T Astragalus hamosus L.; CM; 2, 3; c

P Colutea arborescens L.; CM; 4, 5; a

P Coronilla emerus L. ssp. emeroides Boiss. et Spruner; IM; 2, 4, 5; a, c

T C. scorpioides (L.) Koch; CM; 2, 5; a, c

Ch Dorycnium hirsutum (L.) Ser.; CM; 2 ; a, c, f

T Lathyrus aphaca L.; JEUM; 2, 4, 5; a, c

T L. sphaericus Retz.; JEUM; 3; a, c

$\mathrm{T} \quad$ L. cicera L.; CM; 2; c

$\mathrm{T} \quad$ Lens nigricans (M. Bieb.) Godr.; CM; 2; c

Ch Lotus cytisoides L.; CM; 1, 2, 3, 4, 5; f

T Medicago arabica (L.) Huds.; ŠR; 1, 3; c

T M. coronata (L.) Bartal.; CM; 1, 2; c

H M. falcata L.; EAF; 3; c

T M. littoralis Rohde ex Loisel.; CM; 1, 2, 3, 4, 5; c

T M. lupulina L.; ŠR; 2, 4, 5; C

T M. minima (L.) Bartal.; ŠR; 1, 2, 3, 4, 5; a, b, c

T M. orbicularis (L.) Bartal.; CM; 1, 2, 3; c, d

H M. sativa L. ssp. sativa; ŠR; 3; C 
T Melilotus indica (L.) All.; CM; 3, 4, 5; c

H M. officinalis (L.) Lam.; IM; 3, 5; c, d

T Ononis ornithopodioides L.; CM; 2; c

$\mathrm{H} \quad$ O. pusilla L.; JEUM; 2, 5; a, c

T O. reclinata L.; CM; 2 ; $\mathrm{C}$

T Pisum satioum L. ssp. elatius (M. Bieb.) Asch. et Graebn.; JEUM; 4, 5; c

T Securigera cretica (L.) Lassen (= Coronilla cretica L.); IM; 2, 3; C

$\mathrm{T} \quad$ Trifolium angustifolium L.; CM; 5; a, c

$\mathrm{T} \quad$ T. arvense L.; EAF; 2, 5; c

T T. campestre Schreber; ŠR; 1, 2, 3, 4, 5; a, c

T T. scabrum L.; CM; 1, 2, 3, 4, 5; a, b, c, d, f

T T. stellatum L.; CM; 1 ; C

T T. striatum L.; EAF; 2, 3, 4; c, d

T T. subterraneum L.; MA; $2 ; \mathrm{d}$

T T. suffocatum L.; CM; $1 ; \mathrm{f}$

T T. tomentosum L.; CM; 2; c

T Trigonella esculenta Will; MA; 1, 2, 4; c, d

T T. monspeliaca L.; CM; 2; C

$\mathrm{T} \quad$ Vicia angustifolia L. ssp. angustifolia; EF; 2, 3, 4, 5; C

T V. parviflora Cav.; CM; 2; c

T V. tenuissima (M. Bieb.) Schinz et Thell. (=V. gracilis Loisel.); JEUM; 2; c

T V. villosa Roth. ssp. varia (Host) Corb.; IEP; 4; c

\section{Fagaceae}

P Quercus ilex L.; CM; 3, 4, 5; a, b

P Q. pubescens Willd.; JEUP; 3; c

\section{Fumariaceae}

T Fumaria capreolata L.; MA; 2, 4, 5; a, d

$\mathrm{T} \quad$ F. officinalis L.; ŠR; 1, 3, 4, 5; d

\section{Gentianaceae}

T Centaurium erythraea Rafn; ŠR; 5; c

\section{Geraniaceae}

T Erodium cicutarium (L.) L'Hér.; ŠR; 1 , 2; d

T E. malacoides (L.) L'Hér.; CM; 1, 3; d

$\mathrm{T}$ Geranium columbinum L.; EAF; 1, 2; C

T G. lucidum L.; MA; 2; c

$\mathrm{T} \quad$ G. molle L. ssp. molle; ŠR; 4 ; c

T G. molle L. ssp. brutium (Gasparr.) Graebn.; IM; 3; c

T G. purpureum Vill.; JEUM; 1, 2, 4, 5; a, b, g

T G. rotundifolium L.; EAF; 1, 2, 3, 4; c

\section{Lamiaceae}

T Acinos arvensis (Lam.) Dandy; EF; 2; c

T Ajuga chamaepitys (L.) Schreb.; CM; 2; c

$\mathrm{H} \quad$ Calamintha nepetoides Jord.; JEUP; 1, 3, 4; d

$\mathrm{T} \quad$ Lamium amlexicaule L.; EAF; 1,$2 ; \mathrm{d}, \mathrm{g}$

$\mathrm{H} \quad$ Marrubium incanum Desr.; ILAP; 1 ; d

Ch Prasium majus L.; CM; 2, 4, 5; a

$\mathrm{H}$ Salvia bertolonii Vis.; ILJAE; 1, 2, 3, 5; c 
T Sideritis romana L.; CM; 1, 2, 5; C

Ch Teucrium chamaedrys L.; JEUP; 2, 5; a, b, c

Ch T. polium L. ssp. capitatum (L.) Arcang; MP; 2, 5; a, b, c

Linaceae

$\mathrm{H} \quad$ Linum bienne Mill.; MA; 4;

$\mathrm{T} \quad$ L. strictum L. ssp. corymbulosum (Rchb.) Riony; MP; 5; c

Malvaceae

$\mathrm{H} \quad$ Malva sylvestris L.; ŠR; 1, 2, 3, 4; d

Moraceae

P Ficus carica L.; CM; 4;

\section{Oleaceae}

P Olea europaea L. (incl. O. europaea L. var. oleaster (Hoffm. et Link) Fiori); N; 3, 5; a, c

P Phillyrea latifolia L.; CM; 2, 4, 5; a, b

\section{Orobanchaceae}

T Orobanche nana Noë; JEUP; 1 , 3; c

\section{Papaveraceae}

T Papaver rhoeas L.; ŠR; 2 ; d

\section{Plantaginaceae}

H Plantago altissima L.; JEUM; 1, 2, 3; c, d

T P. coronopus L. ssp. commutata (Guss.) Pilger; MP; 1, 2; e

H P. lanceolata L.; ŠR; $1,2,3,4 ; b, \mathrm{~d}$

\section{Plumbaginaceae}

$\mathrm{H} \quad$ Limonium cancellatum (Bernh. ex Bertol.) O. Kuntze; ILAP; 2, 3, 4, 5; f; end; spr

$\mathrm{H} \quad$ L. narbonense Mill.; CM; 1, 3, 4, 5; e

\section{Polygonaceae}

T Fallopia convolvulus (L.) Á. Löve; CIRC; 2; d

T Polygonum aviculare L.; ŠR; 5; d

$\mathrm{H} \quad$ Rumex pulcher L.; JEUP; 2, 3; d

\section{Primulaceae}

T Anagallis arvensis L.; ŠR; 1, 2, 3, 4, 5; b, c, d

$\mathrm{T} \quad$ A. coerulea Schreb.; ŠR; 2,5 ; c

$\mathrm{T}$ Asterolinon linum-stellatum (L.) Duby; CM; 2, 5; c

G Cyclamen repandum Sibth. et Sm.; EUM; 2; a, b

\section{Ranunculaceae}

P Clematis flammula L.; CM; 2, 3, 4, 5; a

\section{Rhamnaceae}

P Paliurus spina-christi Mill.; ILJEU; 1; a

\section{Rosaceae}

$\mathrm{T}$ Aphanes arvensis L.; EF; 1, 2; C

P Prunus mahaleb L.; JEUP; 2; c

P Rubus ulmifolius Schott; MA; 2, 3; c, d

\section{Rubiaceae}

$\mathrm{T}$ Galium aparine L.; ŠR; 1, 2, 3, 4, 5; b, d

$\mathrm{H} \quad$ G. corrudifolium Vill.; JEUM; 2, 5; a, c 
T G. murale (L.) All.; CM; 2; c

P Rubia peregrina L.; CM; 2, 4, 5; a, b, c

T Sherardia arvensis L.; ŠR; $1,2,3,4,5$; c

T Valantia muralis L.; CM; 1, 2, 4, 5; f

\section{Santalaceae}

P Osyris alba L.; CM; 3, 4, 5; a

\section{Scrophulariaceae}

$\mathrm{T} \quad$ Linaria simplex (Willd.) DC.; CM; 5; c

$\mathrm{H} \quad$ Verbascum pulverulentum Vill.; JT; 3; c

$\mathrm{T} \quad$ Veronica arvensis L.; EAF; 1, 2, 3, 5; c

T V. cymbalaria Bodard; JEUM; $4 ; \mathrm{g}$

T V. persica Poir.; ŠR; 1, 2; d

T V.polita Fr.; EAF; 1,$4 ; \mathrm{c}, \mathrm{d}$

T V. praecox All.; EUM; 1 ; c

\section{Tamaricaceae}

$\mathrm{P} \quad$ Tamarix dalmatica Baum; ZM; 3; f

\section{Ulmaceae}

$\mathrm{P} \quad$ Celtis australis L.; JEUM; 3; C

\section{Urticaceae}

$\mathrm{H} \quad$ Parietaria judaica L.; JEUM; 1, 2, 3, 4, 5; b, d

T Urtica urens L.; ŠR; 1, 2, 3; d

\section{Valerianaceae}

$\mathrm{T} \quad$ Valerianella muricata (Stiven ex M.Bieb.) J.W. Loudon; CM; 2; C

\section{Verbenaceae}

$\mathrm{P} \quad$ Vitex agnus-castus L.; CM; 2; $\mathrm{f}$

\section{Violaceae}

T Viola kitaibeliana Schultes; CM; 2; c

\section{LILIATAE}

\section{Araceae}

G Arum italicum Mill.; CM; 1, 2, 3, 4, 5; a, b, d

\section{Cyperaceae}

$\mathrm{H} \quad$ Carex distachya Desf.; CM; 4; c

G C. divisa Huds.; MA; 3; c; spr; EN

$\mathrm{H} \quad$ C. divulsa Stokes; ŠR; 4 ; c

H C. extensa Gooden.; ŠR; 2, 3; e; spr; EN

G C. flacca Schreb. ssp. serrulata (Biv.) Greuter; ŠR; 5; a, c

$\mathrm{H} \quad$ C. hallerana Asso; JEUM; 2; C

$\mathrm{H} \quad$ Schoenus nigricans L.; ŠR; 4, 5; $\mathrm{f}$

\section{Dioscoreaceae}

G Tamus communis L.; JEUM; 2, 4, 5; a, b

\section{Iridaceae}

G Romulea bulbocodium (L.) Seb. et Mauri; CM; 2, 4; c

\section{Juncaceae}

H Juncus acutus L.; MA; 3; f

G J. maritimus Lam.; ŠR; 3, 4; e, f 


\section{Liliaceae}

G Allium commutatum Guss.; CM; 3, 4, 5; f

G A. roseum L.; CM; 3; c

G A. subhirsutum L.; CM; 2, 3, 4, 5; a, b

G Asparagus acutifolius L.; CM; 1, 2, 3, 4, 5; a, b, c

G Asphodelus aestivus Brot.; CM; 1, 2, 4, 5; c

G Muscari comosum (L.) Mill.; JEUM; 2, 3, 4, 5; c

G M. neglectum Guss. ex Ten.; JEUM; 2; c

G Ornithogalum pyramidale L.; JEUM; 5; c

P Smilax aspera L.; CM; 2, 4, 5; a, b, c

\section{Poaceae}

$\mathrm{H}$ Anthoxanthum odoratum L.; EAF; 2; C

T Avena barbata Pott ex Link; JEUP; 1, 2, 3, 4, 5; d

T A. sterilis L.; JEUP; 4; d

T Brachypodium distachyon (L.) P.Beauv.; CM; 2; c

$\mathrm{H} \quad$ B. pinnatum (L.) P. Beauv. ssp. rupestre (Host) Schubl. et M. Martens; AN; 4 ; b, c

$\mathrm{H} \quad$ B. retusum (Pers.) P. Beauv.; CM; 2, 5; a, b, c, d, f

T Briza maxima L.; CM; 2 ;

H Bromus erectus Huds. ssp. condensatus (Hack.) Asch. et Graebn.; JEUM; 2, 3, 4, 5; C

T B. hordaceus L. ssp. molliformis (Lloyd) Maire et Weiller; JEUM; 1, 2, 4; c

T B. madritensis L.; MA; 3, 5; d

T B. sterilis L.; ŠR; 1, 2, 3, 4; d

$\mathrm{H} \quad$ Chrysopogon gryllus (L.) Trin.; MP; 5; C

G Cynodon dactylon (L.) Pers.; ŠR; 1, 2, 4, 5; d, f

T Cynosurus echinatus L.; JEUM; 2; C

H Dactylis glomerata L. ssp. glomerata; EAF; 5; c

H D. glomerata L. ssp. hispanica (Roth) Nyman; CM; 1, 2, 3, 4; c

T Desmazeria marina (L.) Druce; MA; 1, 2, 4, 5; f; spr; VU

$\mathrm{T} \quad$ D. rigida (L.) Tutin; MA; $1,2,3,4$, 5; C

$\mathrm{H}$ Dichanthium ischaemum (L.) Roberty; JEUM; 4; C

G Elymus pycnanthus (Godr.) Melderis; CM; 1, 2, 3, 4, 5; f; NT

G E. repens (L.) Gould; ŠR; 3; c, d

$\mathrm{H} \quad$ Festuca pratensis Huds.; ŠR; 3, 4; c

$\mathrm{H} \quad$ F. pseudovina Hack. ex Wiesb.; IEP; 2; c

H Helictotrichon convolutum (C. Presl) Henrard; ZM; 2, 4, 5; C

$\mathrm{T} \quad$ Hordeum murinum L. ssp. leporinum (Link) Arcang.; CM; 1, 2, 3, 5; d

$\mathrm{T} \quad$ Lagurus ovatus L.; CM; 1, 2, 3, 4; b, d

$\mathrm{H} \quad$ Lolium perenne L.; EF; 1 ; d

$\mathrm{T} \quad$ L. rigidum Gaudin ssp. lepturoides (Boiss.) Sennen et Mauricio; IM; 3, 4, 5; d, e

T L. strictum L. ssp. strictum; JEUM; 1, 3, 4; c

T Lophochloa cristata (L.) Hyl.; MA; 1, 2, 5; c

$\mathrm{H} \quad$ Melica ciliata L.; EAF; 2, 3, 4; c, g; LC

T Parapholis incurva (L.) C.E.Hubb.; MA; 1, 2, 3, 4, 5; e, f; spr; VU

H Poa bulbosa L.; EAF; 1, 2, 5; C

H P. pratensis L.; ŠR; 3; c

$\mathrm{H} \quad$ Puccinellia festuciformis (Host) Parl.; MP; 3; e

T Vulpia ciliata Dumort.; JEUM; 2; c 


\section{ANALYSIS OF THE FLORA}

\section{Taxonomical analysis}

On the five uninhabited islets situated in the vicinity of village of Pakoštane, there were 257 vascular flora taxa (225 species and 32 subspecies), which were classified into 169 genera and 54 families (Tab.1). This is the first recording of the taxa for these islets.

The largest number of taxa was found on Veliki Školj (159), followed by St. Justina (118), Žavinac Veliki (115), Žavinac Mali (105) and Babuljaš (95) (Tab. 2).

As expected, the largest number of taxa is recorded for V. Školj (159). It has the largest area $\left(0,055 \mathrm{~km}^{2}\right)$, the highest point above the sea level $(27 \mathrm{~m})$ and it is moderately exposed to anthropogenic influence (goat grazing). The other four islets are smaller $(0.008$ $-0.028 \mathrm{~km}^{2}$ ), and are lower lying, which makes their vegetation completely exposed to salinization. St. Justina $\left(0,013 \mathrm{~km}^{2}\right)$ is one-half the size of Žavinac Veliki $\left(0,028 \mathrm{~km}^{2}\right)$ but has the largest number of taxa (118 vs. 115). This is due to the greater human influence: a small olive grove has been recently planted on St Justina creating a new type of habitat and resulting in an increase in the richness of the flora.

The results indicate that the richness of the vascular flora of these five islands is influenced more by the diversity of habitats and anthropogenic elements than by the areas of islets.

Families with the highest number of taxa were: Fabaceae (15.56 \% of the total flora), Poaceae (14.00\%) and Asteraceae s.1. (12.06 \%). Their contribution to the total number of taxa was $42 \%$ of the total flora (Tab. 3). These families are also predominant on other Dalmatian islands and islets: Lokrum, Bobara and Mrkan (Hećımović, 1982), Žirje (PANDŽA, 2003), Ist and Škarda (Milović \& PANDžA, 2010), Obonjan (Milović, 2004a), Supetar (JASPRICA \& RuŠČIĆ, 2013) etc.

\section{Analysis of the life forms}

As expected, the proportion of therophytes in the flora of the investigated islets was high (49.42\%) (Fig. 2). This was also shown for each particular islet (Tab. 4).

The proportion of therophytes ranges from $41.53 \%$ on St. Justina to as much as $61.04 \%$ on Babuljaš. Therophytes are also the predominant life form in other Dalmatian islets'

Tab. 1. Taxonomical analysis

\begin{tabular}{|l|c|c|c|c|c|}
\hline \multirow{2}{*}{ Taxa } & Pterydophyta & Gymnospermae & \multicolumn{2}{|c|}{ Angiospermae } & \multirow{2}{*}{ Total } \\
\cline { 3 - 5 } & & & Dicotyledones & Monocotyledones & \\
\hline Families & 1 & 2 & 44 & 7 & 54 \\
\hline Genera & 1 & 3 & 130 & 48 & 169 \\
\hline Species & 1 & 3 & 173 & 9 & 225 \\
\hline Subspecies & - & 2 & 21 & 57 & 257 \\
\hline $\begin{array}{l}\text { Species and } \\
\text { subspecies }\end{array}$ & 1 & 5 & 194 & 22.18 & 100 \\
\hline$\%$ & 0.39 & 1.95 & 75.48 & & 32 \\
\hline
\end{tabular}


Tab. 2. Number of taxa compared with numbers on other uninhabited Dalmatian islets (with surface areas less than one $\mathrm{km}^{2}$ ).

\begin{tabular}{|c|c|c|c|}
\hline Island/Islet & Area $\left(\mathrm{km}^{2}\right)^{*}$ & No. of taxa & References \\
\hline Obonjan & 0.550 & 230 & Milović (2004a) \\
\hline Palagruža & 0.286 & 220 & Pavletić (1983) \\
\hline Mrkan & 0.197 & 179 & Hećimović (1982) \\
\hline Zečevo (Hvar) & 0.11 & 216 & SKELIN et al. (2014) \\
\hline Kozina & 0.063 & 105 & PANDŽA et al. (2011) \\
\hline Veliki Školj & 0.055 & 159 & this study \\
\hline Hrbošnjak (Murter) & 0.050 & 84 & PANDŽA (2002) \\
\hline Samograd & 0.043 & 71 & PANDŽA (2003) \\
\hline Supetar (Cavtat) & 0.039 & 173 & JASPRICA \& RUŠČIĆ (2013) \\
\hline St Andrija & 0.036 & 160 & JASPRICA et al. (2006) \\
\hline Veliki Žavinac & 0.028 & 115 & this study \\
\hline Dražemanski Mali & 0.027 & 81 & PANDŽA (2002) \\
\hline Sustipanac & 0.0125 & 123 & PANDŽA (2002) \\
\hline St Justina & 0.0125 & 118 & this study \\
\hline Kamenjak (Ist) & 0.0119 & 34 & MiLović \& PANDŽA (2010) \\
\hline Tužbina & 0.0119 & 62 & PANDŽA (2002) \\
\hline Mali Žavinac & 0.0115 & 105 & this study \\
\hline Benušić (Ist) & 0.006 & 33 & Milović \& PANDŽA (2010) \\
\hline Babuljaš & $<0.001$ & 95 & this study \\
\hline
\end{tabular}

* According to Duplančić Leder et al. (2004)

Tab. 3. List of families with $\geq 10$ taxa.

\begin{tabular}{|l|c|c|}
\hline Family & No. of taxa & \% of total flora \\
\hline Fabaceae & 40 & 15.56 \\
\hline Poaceae & 36 & 14.00 \\
\hline Asteraceae s.l. & 31 & 12.06 \\
\hline Asteraceae s.s. & $(13)$ & $(5.06)$ \\
\hline Cichoriaceae & $(18)$ & $(7.00)$ \\
\hline Caryophyllaceae & 15 & 5.84 \\
\hline Brassicaceae & 11 & 4.28 \\
\hline Lamiaceae & 10 & 3.89 \\
\hline Other families (47) & 114 & 44.37 \\
\hline Total & 257 & 100.00 \\
\hline
\end{tabular}

flora (PANDŽA, 2002; JASPRICA \& RuščIĆ, 2013; SKELIN et al., 2014), indicative of the dry Mediterranean climate. Life form spectra in the flora of the investigated islets do not significantly differ from those recorded for other Dalmatian uninhabited islets (MiLović, 2004a; Bogdanović\&Mitić, 2003; JAsprica et al., 2006; PANDŽA et al., 2011; SKelin et al., 2014). 
Tab. 4. Life forms in the floras of the islets researched.

\begin{tabular}{|l|c|c|c|c|c|c|}
\hline Islets & Therophyta & $\begin{array}{c}\text { Hemicrypto- } \\
\text { phyta }\end{array}$ & Geophyta & Chamaephyta & Phanerophyta & $\begin{array}{c}\text { Total } \\
\text { flora } \\
\end{array}$ \\
\cline { 2 - 6 } & $\begin{array}{c}\text { No. of taxa } \\
(\%)\end{array}$ & $\begin{array}{c}\text { No. of taxa } \\
(\%)\end{array}$ & $\begin{array}{c}\text { No. of taxa } \\
(\%)\end{array}$ & $\begin{array}{c}\text { No. of taxa } \\
(\%)\end{array}$ & $\begin{array}{c}\text { No. of taxa } \\
(\%)\end{array}$ & \\
\hline Babuljaš & $58(61.04)$ & $19(20.00)$ & $6(6.32)$ & $6(6.32)$ & $6(6.32)$ & $95(100)$ \\
\hline V. Školj & $92(57.86)$ & $32(20.13$ & $12(7.55)$ & $10(6.29)$ & $13(8.17)$ & $159(100)$ \\
\hline St. Justina & $49(41.53)$ & $38(32.20)$ & $11(9.32)$ & $9(7.63)$ & $11(9.32)$ & $118(100)$ \\
\hline Žavinac M. & $45(42.86)$ & $28(26.67)$ & $13(12.38)$ & $7(6.67)$ & $12(11.42)$ & $105(100)$ \\
\hline Žavinac V. & $55(47.83)$ & $25(21.74)$ & $12(10.43)$ & $10(8.70)$ & $13(11.30)$ & $115(100)$ \\
\hline
\end{tabular}

\section{Phytogeographical analysis}

As the region of this research belongs to the typical Mediterranean climate, the phytogeographical analysis results (Tab. 6) were as expected: plants of the Mediterranean floral element are the most common (50.19\%). They are followed by widespread $(20.23 \%)$ and South European plants (17.90\%). Such a predominance of Mediterranean plants was also recorded in some other Dalmatian islands' flora (e.g. Milović, 2004a, PANDžA et al., 2011).

Although they are situated only a small distance (less than $0.6 \mathrm{~km}$ ) from the mainland (Fig. 1), anthropogenic influence on the investigated islets is insignificant, with the exception of Veli Škoj (grazing goats) and St. Justina (a small olive grove). Therefore, the poor representation of alien plants $(0.78 \%$ of the total flora) was expected. Low representation of suitable anthropogenic habitats and the exposure of the islets to salinization are the main limiting factors for the immigration of adventive plants. Only two of them were found, both on islet of St. Justina. Olive trees (Olea europaea) have recently been brought into cultivation. The neophyte taxon Conyza sumatrensis has been accidentally brought. This taxon is currently spreading invasively throughout Dalmatia, south Croatia (Milović, 2004b).

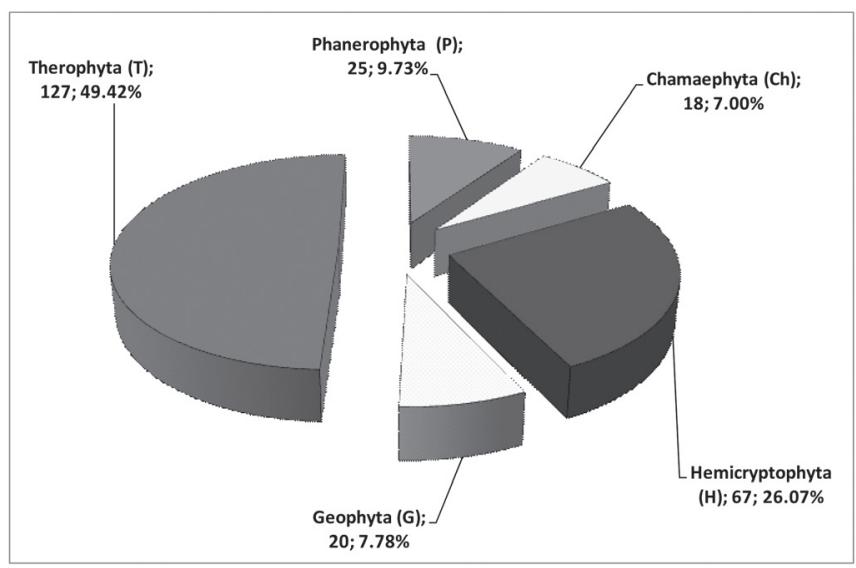

Fig. 2. Spectrum of life forms in the flora on the all investigated islets. 
Tab. 6. Floral elements analysis of the flora of the islets near Pakoštane.

\begin{tabular}{|l|c|c|}
\hline Geoelements & No. of taxa & $\%$ \\
\hline MEDITERRANEAN - MED & 129 & 50.19 \\
\hline SOUTH EUROPEAN - SEU & 46 & 17.90 \\
\hline ATLANTIC PLANTS - AN & 1 & 0.39 \\
\hline EAST EUROPEAN-PONTIC - IEP & 2 & 0.78 \\
\hline EUROPEAN - EF & 4 & 1.56 \\
\hline EUROASIATIC - EAF & 19 & 7.39 \\
\hline CIRCUM-HOLARCTIC - CIRC & 2 & 0.78 \\
\hline WIDESPREAD PLANTS - ŠR & 52 & 20.23 \\
\hline CULTURAL \& ADVENTIVE - N & 2 & 0.78 \\
\hline TOTAL & 257 & 100.00 \\
\hline
\end{tabular}

In the flora of the investigated region, four endemic, eight endangered and eight strictly protected taxa were found. Three endemic taxa were found on dry rocky grasslands (Vincetoxicum hirundinaria ssp. adriaticum, Carduus micropterus ssp. micropterus and Aurinia sinuata) while Limonium cancellatum grows on rocks in the halophytic zone. There were two endangered (Carex divisa and C. extensa) and two vulnerable (Desmazeria marina and Parapholis incurva) taxa, and they are also found in the coastal zone which is exposed to salinization.

\section{CONCLUSION}

In this study, 257 plant taxa (species and subspecies) are recorded for the first time for the five uninhabited islets near Pakoštane, North Dalmatia. Due to the small surface areas and some particular ecological conditions, the flora of islets showed characteristics similar to those of some other Dalmatian islands. These results contribute to the broadening of our knowledge on the vascular plant diversity of the uninhabited Adriatic islands.

\section{ACKNOWLEDGEMENTS}

We would like to thank the two anonymous reviewers for their constructive comments on an earlier version of this manuscript.

Received May 11, 2014

\section{REFERENCES}

Anonymous, 1992: Council Directive 92/43/EEC of 21 May 1992 on the conservation of natural habitats and wild fauna and flora. Annex I-IV. Council oft he European Communities.

Anonymous, 2007: Državni program zaštite i korištenja malih, povremeno nastanjenih i nenastanjenih otoka i okolnog mora. Ministry of Maritime Affairs, Transport and Infrastructure of Republic of Croatia.

Anonymous, 2013a: Zakon o zaštiti prirode. NN 80/2013. 
Anonymous, 2013b: Pravilnik o strogo zaštićenim vrstama. NN 144/2013, Prilog I.

Bertović, S., 1975: Prilog poznavanju odnosa klime i vegetacije u Hrvatskoj. Prir Istraž 41, JAZU, Zagreb.

Bogdanović, S. \& Mitić, B., 2003: The flora of the volcanic island of Brusnik (central Dalmatia, Croatia). Acta Bot. Croat. 62(2), 103-113.

Bonnier, G., 1911-1935: Flore compléte illustrée en couleurs de France, Suisse et Belgique. Neuchatel, Paris et Bruxelles.

Doмac, R., 1994: Flora Hrvatske. Priručnik za određivanje bilja. Zagreb.

Duplančić Leder, T., Ujević, T. \& ČAla, M., 2004: Coastline lengths and areas of islands in the Croatian part of the Adriatic Sea determined from the topographic maps at the scale of 1:25000. Geoadria 9(1), $5-32$.

FIorI, A., 1923-1929: La Nuova Flora Analitica d'Italia. I-II. Firenze.

HAyeK, A., 1924-1933: Prodromus florae Peninsulae Balcanicae. I-III. Dahlem bei Berlin.

Hećimović, S., 1982: Flora otoka Lokruma, Bobare i Mrkana. Acta Bot. Croat. 41, 155-170.

HeGI, G., 1936-1987: Illustrierte Flora von Mittel-Europa (2nd ed.), München.

HoRvat, I., 1949: Nauka o biljnim zajednicama. Nakladni zavod Hrvatske, Zagreb.

Honvatić, S., 1963: Vegetacijska karta otoka Paga s općim pregledom vegetacijskih jedinica Hrvatskog primorja. Zagreb. Prir. Istraž. Jugosl. Akad. 33. Acta biologica 4. Zagreb.

Horvatić, S., Ilijanić, Lj. \& Marković-Gospodarić, Lj., 1967-1968: Biljni pokrov okolice Senja. Senjski zbornik 3, 298-322.

Horvatić, S. \& TrinajStić, I. (eds.), 1967 - 1981: Analitička flora Jugoslavije 1. Šumarski fakultet Sveučilišta u Zagrebu, Sveučilišna naklada Liber, Zagreb.

JAsprica, N. \& Ruščić, M., 2013: Flora i vegetacija otočića Supetra (Cavtat, južna Hrvatska). Hrvatska misao (Sarajevo) 46, 111-134.

Jasprica, N., Kovačić, S. \& Ruščić, M., 2006: Flora and vegetation of Sveti Andrija Island, Southern Croatia. Nat. Croat., 15(1-2), 27-42.

JAVORKA, S. \& CSÁPODY, V., 1975: Iconographia florae partis Austro-orientalis Europae centralis. Akadémiai Kiadó, Budapest.

Milović, M., 2004a: Flora of the island of Obonjan (Šibenik archipelago, Croatia). Nat. Croat. 13 (3), $213-230$.

Mirović, M., 2004b: Naturalised species from the genus Conyza Less. (Asteraceae) in Croatia. Acta Bot. Croat. 63 (2), 147-170.

Milović, M. \& PANDžA, M., 2010: Vaskularna flora otoka Ista i Škarde s pripadajućim otočićima i hridima. In: FARIČrć, J. (ed.): Otoci Ist i Škarda. Sveučilište u Zadru, Zadar, 149-186.

NiкoLIć, T. (ed.), 2014a: Flora Croatica baza podataka / Flora Croatica Database. On-Line, URL: http:// hirc.botanic.hr/fcd. Botanički zavod s Botaničkim vrtom, Prirodoslovno-matematički fakultet, Sveučilište u Zagrebu. [last accessed on May 20, 2014].

Niкоціс́, T. (ed.), 2014b: Crvena knjiga vaskularne flore Hrvatske / Red Dana Book of Vascular Flora of Croatia. On-Line URL: http://hirc.botanic.hr/fcd/CrvenaKnjiga. Botanički zavod s Botaničkim vrtom, Prirodoslovno-matematički fakultet, Sveučilište u Zagrebu. [last accessed on May 20, 2014].

Niкоlı́́, T. \& Topıć, J. (ed.), 2005: Crvena knjiga vaskularne flore Hrvatske. Ministarstvo kulture. Državni zavod za zaštitu prirode. Zagreb.

Nikolić, T., Antonić, O., Alegro, A.L., Dobrović, I., Bogdanović, S., Liber, Z. \& Rešetnik, I., 2008: Plantspecies diversity of Adriatic islands: An introductory survey. Plant Biosystems 142(3), 435-445.

Nikolić, T., Mitić, B., Ruščıć, M. \& Milašınović, B., 2014: Diversity, knowledge and spatial distribution of the vascular flora of Croatia. Plant Biosystems. 148 (4), 591-601.

Pignatti, S., 1982: Flora d'Italia. I-III. Edagricole. Bologna.

PANDŽA, M., 2002: Flora of the small islands of Murter. Nat. Croat. 11(1), 77-101.

PANDŽA, M., 2003: Flora of the island of Žirje and small islands around it (eastern Adriatic coast, Croatia). Acta Bot. Croat. 62(2), 115-139.

PAndžA, M., Milović, M., KRPina, V. \& Tafra, D., 2011: Vascular flora of the Vrgada islets (Zadar archipelago, eastern Adriatic). Nat. Croat. 20(1), 97-116.

PAvletić, Zı., 1983: Pregled flore i vegetacije nekih manjih srednjodalmatinskih otoka i otočića. Zbornik Roberta Visianija Šibenčanina, povremena izdanja Muzeja grada Šibenika 10, 315-328.

Penzar, B. \& Penzar, I.: 1979-1980: O položaju i uzrocima ekstrema u godišnjem hodu oborine u Hrvatskoj. Geografski glasnik 41-42, 27-48. 
Penzar, B. \& Penzar, I., 1981: O položaju i uzrocima ekstrema u godišnjem hodu oborine u Hrvatskoj. Geografski glasnik 43: 27-49.

Raunkiaer, C., 1934: The life forms of plants and statistical plant geography. The Clarendon Press, Oxford, pp. 632.

Skelin, M., Ljubičić, I., Skelin, I., Vitasović Kosić, I. \& Bogdanović, S., 2014: The Flora of Zečevo (Hvar Archipelago, Croatia). Agriculturae Conspectus Scientificus 79(2), 85-91.

STRAŽIČIĆ, N., 1987: Prirodno-geografske značajke kao poticajni i ograničavajući faktori razvoja jadranskih otoka. Pomorski zbornik 25, 39-55.

ŠEgota, T. \& Filırčıć, A., 2003: Köppenova podjela klima i hrvatsko nazivlje. Geoadria 8(1), 17-37.

Topić, J. \& Vukelić, J., 2009: Priručnik za određivanje kopnenih staništa u Hrvatskoj prema direktivi o staništima EU. Državni zavod za zaštitu prirode, Zagreb.

TrinAJSTIĆ, I. (ed.), 1975-1986: Analitička flora Jugoslavije 2(1-4). Institut za botaniku Sveučilišta u Zagrebu, Sveučilišna naklada Liber, Zagreb.

Tutin, T.G., Burges, N.A., Chater, A.O., Edmondson, J.R.E., Heywood, V.H., Moore, D.M., Valentine, D.H., Walters, S.M. \& Webb, D.A. (eds.), 1993: Flora Europaea 1 (2nd edn.), Cambridge University press. Cambridge.

Tutin, T.G., Heywood, V.H., Burges, N.A., Moore, D.M., Valentine, D.H., Walters, S.M. \& Webb, D.A. (eds.), 1968-1980: Flora Europaea 2-5. Cambridge University press. Cambridge.

\section{SAŽETAK}

\section{Flora otočića ispred Pakoštana (Dalmacija, Hrvatska)}

\section{Pandža \& M. Milović}

Vaskularna flora nenaseljenih i slabo naseljenih otočića i hridi hrvatskog otočnog arhipelaga je slabo istražena. Tijekom 2004. i 2012. provedena su terenska istraživanja flore na pet nenaseljenih otočića ispred Pakoštana (zadarski arhipelag, sjeverna Dalmacija): Babuljaš, Veliki Školj, Sveta Justina, Mali i Veliki Žavinac. Zbog male površine i male nadmorske visine, vegetacija istraživanih otočića gotovo je u cijelosti izložena zaslanjivanju. Značajnijem antropogenom utjecaju izloženi su samo Sv. Justina (maslinik i crkvica) i Veliki Škoj (ispaša koza). Za pet istraživanih otočića utvrđeno je ukupno 257 svojti vaskularne flore. Flora Velikog Školja ima 159 svojti, Sv. Justine 118, Velikog Žavinca 115, Malog Žavinca 105 i Babuljaša 95 svojti. Zabilježeni broj svojti ne odstupa značajno u odnosu na broj svojti koji je zabilježen za druge nenaseljene otočiće u Dalmaciji.

Rezultati taksonomske, fitogeografske i ekološke analize flore su očekivani s obzirom na fitogeografski položaj i tipičnu mediteransku klimu i u skladu su s rezultatima utvrđenim za floru drugih dalmatinskih otočića. Porodice s najvećim brojem svojti su Fabaceae (15,56\%), Poaceae (14,00\%) i Asteraceae s.l. (12,06\%). Terofiti su najzastupljeniji životni oblik (49,42\%), a među flornim elementima najzastupljenije su mediteranske biljke (50,19\%), koje su približno jednako zastupljene u flori svih pet otočića $(47,62-53,04 \%)$. U flori istraživanih otočića su značajno zastupljene svojte od posebnog interesa: zabilježena su četiri endema, osam ugroženih te osam strogo zaštićenih svojti. Od neofita, na Sv. Justini je zabilježena samo vrsta Conyza sumatrensis dok na ostalim otočićima neofita nije bilo. Izostanak većeg broja stranih vrsta posljedica je slabo izraženog antropogenog utjecaja i izostanka odgovarajućih staništa pogodnih za njihovo useljavanje. 